\title{
HydroTherapy in der Wundversorgung
}

\section{Effektives Débridement ohne Skalpell}

") Das autolytische Débridement - im Rahmen der HydroTherapy mit dem Wundkissen HydroClean ${ }^{\circledR}$ vorgenommen - ist eine sanfte und schonende Alternative zum chirurgischen Débridement. Das supraabsorbierende Wundkissen verfügt als einzige Wundauflage über einen einzigartigen SaugSpül-Mechanismus. HydroClean ${ }^{\circledR}$ führt der Wunde durch kontinuierliches Spülen mit Ringerlösung Feuchtigkeit zu, löst selektiv Nekrosen, Exsudat, Fibrin und Bakterien, nimmt diese auf und bindet sie sicher im Kern. Proteolytische Enzyme und Phagozyten werden aktiviert. Durch die Beseitigung von Keimreservoiren und die
Verringerung der Keimkonzentration wird das Infektionsrisiko gesenkt, die Beseitigung von Gewebebarrieren und die Reduzierung von Matrixmetalloproteasen (MMP) fördert die Granulation und Epithelisierung der Wunde. Auf diese Weise schafft das Wundkissen optimale Wundverhältnisse für eine effiziente Wundheilung, die in der Granulations- und Epithelisierungsphase mit dem mit Hydrogel beschichteten Schaumverband HydroTac ${ }^{\circledR}$ weiter unterstützt werden kann.

Das autolytische Débridement mit dem Wundkissen verfüge in der praktischen Anwendung über eine Reihe weiterer Vorteile, berichtete Burkhard
Jendrusch, Krankenpfleger und geprüfter Wundberater aus Westerstede: „HydroClean ${ }^{\circledR}$ kann bis zu drei Tage getragen werden; Silikonstreifen verhindern ein Verkleben mit der Wunde und sorgen für einen schmerzarmen und gewebeschonenden Verbandswechsel; dank einer visuellen Applikationshilfe ist das Kissen, das auch unter Kompression angewendet werden kann, einfach anzulegen."

(koc)

Vortrag „Was wäre, wenn Sie auch ohne Skalpell wirksam débridieren könnten?"; Deutscher Wundkongress Bremen, 11. Mai 2017

(Veranstalter: Paul Hartmann AG)

\section{Volkskrankheit Depression}

\section{Behandlungsoption Internet-Therapie?}

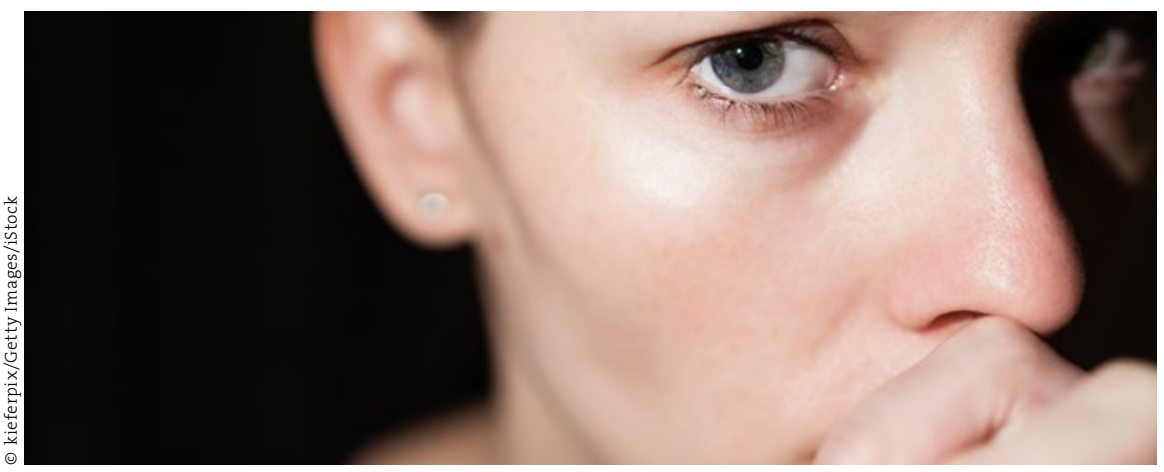

» Depressionen gehören weltweit zu den häufigsten Erkrankungen. In Deutschland erkrankten nach Zahlen des Robert Koch-Instituts mehr als 6\% der Bevölkerung im Laufe eines Jahres daran, berichtete Prof. Kai G. Kahl von der Medizinischen Hochschule Hannover (MHH). Doch die Wartezeit auf einen Therapieplatz beträgt bis $\mathrm{zu}$ sechs Monate. Rasche Hilfe sei erforderlich, auch durch evidenz-basierte Online-Therapieprogramme.

„Grundsätzlich kann neben einer medikamentösen Behandlung auch eine fundierte Psychotherapie helfen", erläuterte Kahl. „Doch während der Wartezeit zwischen Diagnose und Therapiebeginn ist der Patient mit seinem Leidensdruck oft auf sich allein gestellt.“
Man müsse sich von der Vorstellung lösen, dass der Patient dem Behandler immer face-to-face gegenüber sitzen müsse. Ein Teil der Lösung des Problems könnten evidenzbasierte Online-Therapieprogramme wie deprexis ${ }^{\circledR} 24$ sein. Die antidepressive Wirksamkeit wurde Kahl zufolge in acht wissenschaftlichen Studien in Deutschland mit mehreren tausend Patienten nachgewiesen. Es vermindere nachweislich die Beschwerden einer unipolaren Depression oder depressiven Verstimmung.

Wie das Ganze funktioniert, erläuterte Dr. Lotta Winter, Ambulanzzentrum der $\mathrm{MHH} \mathrm{GmbH}$ : Das individualisierte und datenschutzkonforme Programm führt einen interaktiven Dialog mit dem Anwender und lässt sich als Begleiter durch den Alltag überall und jederzeit nutzen. Es bestehe aus zehn Themenkomplexen aus der kognitiven Verhaltenstherapie, wie Verhaltensaktivierung, Achtsamkeit oder Entspannungsübungen. „Ziel ist es, dass der Patient Techniken der Verhaltenstherapie für sich anwendet und lernt, mit der Symptomatik der Depression besser umzugehen", so Winter. Die Begleitung durch einen Arzt oder Psychotherapeuten mittels einer Cockpit-Funktion verbessere den Effekt des Programms. Sie betonte: „Es ist aber kein Ersatz für die Diagnosestellung oder die Behandlung durch einen Arzt oder Psychotherapeuten."

Die Erstattung von Online-Therapieprogrammen ist nicht grundsätzlich für alle Krankenkassen geregelt. „Einen kostenlosen Zugang bietet zum Beispiel die DAK an“, sagte Oliver Kirst, Geschäftsleiter Servier Deutschland. Mit anderen Kassen sei man im Gespräch.

Pressekonferenz „Mentale Gesundheit: Digitale Therapien als neue Chance?"; Deutscher Pflegetag Berlin, 24.03.2017 (Veranstalter: Servier Deutschland $\mathrm{GmbH}$ ) 